\title{
MRI - Mammography 2D/3D Data Fusion for Breast Pathology Assessment
}

\author{
Christian P. Behrenbruch ${ }^{1}$, Kostas Marias ${ }^{1,2}$, Paul A. Armitage ${ }^{1}$, Margaret Yam ${ }^{1}$, \\ Niall Moore $^{3}$, Ruth E. English ${ }^{4}$, J. Michael Brady ${ }^{1}$ \\ ${ }^{1}$ Medical Vision Laboratory (Robotics), Engineering Science, Oxford University, Parks \\ Road, Oxford OX1 3PJ, UK \\ $\{$ cpb,jmb\}@robots.ox.ac.uk, http://www.robots.ox.ac.uk/ mvl \\ ${ }^{2}$ Department of Surgery, Royal Free and University College Medical School, UCL, \\ London NW3 2QG, UK \\ ${ }^{3}$ Magnetic Resonance Imaging Centre, John Radcliffe Hospital, Headley Way, \\ Oxford OX3 9DU, UK \\ ${ }^{4}$ Breast Care Unit, Churchill Hospital, Oxford OX3 7LJ, UK
}

\begin{abstract}
Increasing use is being made of contrast-enhanced Magnetic Resonance Imaging (Gd-DTPA) for breast cancer assessment since it provides $3 \mathrm{D}$ functional information via pharmacokinetic interaction between contrast agent and tumour vascularity, and because it is applicable to women of all ages. Contrast-enhanced MRI (CE-MRI) is complimentary to conventional Xray mammography since it is a relatively low-resolution functional counterpart of a comparatively high-resolution 2D structural representation. However, despite the additional information provided by MRI, mammography is still an extremely important diagnostic imaging modality, particularly for several common conditions such as ductal carcinoma in-situ (DCIS) where it has been shown that there is a strong correlation between microcalcification clusters and malignancy [1]. Pathological indicators such as calcifications and fine spiculations are not visible in CE-MRI and therefore there is clinical and diagnostic value to fusing the high-resolution structural information available from mammography with the functional data acquired from MRI imaging. This paper presents a novel data fusion technique whereby medio-lateral (ML) and cranio-caudal (CC) mammograms (2D data) are registered to 3D contrastenhanced MRI volumes. We utilise a combination of pharmacokinetic modelling, projection geometry, wavelet-based landmark detection and thinplate spline non-rigid registration to transform the coordinates of regions of interest (ROIs) from the 2D mammograms to the spatial reference frame of the contrast-enhanced MRI volume.
\end{abstract}

\section{Introduction}

This paper introduces a system that has been developed to perform data fusion between 2D X-ray mammography and 3D contrast-enhanced MRI of the breast. The objective has been to develop a registration and visualisation framework that makes use of both the high spatial resolution of mammography and the vascular information 
provided by contrast-enhanced MRI. This is particularly useful in situations where small opacities, microcalcifications and fine spiculations, not visible in an MRI scan, cannot be correlated with voxel enhancement.

The principal concept behind this work is the registration of a highly compressed 2D projective representation of the breast with an uncompressed volume-based acquisition. In the case of the MRI acquisition, the patient is lying facedown in the scanner with the breasts resting pendulously and uncompressed in the breast coils. This is entirely different to the situation in mammography, where the patient normally remains upright with the breasts compressed, (one at a time), between two plates. All of the patients in our study had both a medio-lateral (ML) and a cranio-caudal (CC) mammogram, as is required for breast cancer screening in the UK. It is worth noting that the angular separation between the mammogram view directions depends on the woman's size and shape, but typically varies between 45-60 degrees. More importantly, as regards matching, the compression between the two mammograms differs significantly, often by as much as $1 \mathrm{~cm}$.

In order to register the projective X-ray image with the MRI volume, we project the MRI volume in both the CC and the ML direction. However, rather than simply use voxel intensity or approximate T1-correlated X-ray attenuation characteristics [2], we use an enhancement measure based on a two-compartment pharmacokinetic model of contrast uptake [3]. This effectively produces a contrast-based projection that has the visual and structural characteristics of an "enhanced" X-ray mammogram.

The registration technique is outlined in Figure 1. Our registration process actually consists of two separate registrations. The first "partial registration" utilises a curvature measure to correlate boundary points along the film edge of the mammograms with the edge of the volume projection [4]. This curvature measure depends on a good segmentation of the breast edge, which is achieved by an intensitybased search, mathematical morphology for smoothing and spline fitting to produce a smoothed edge profile [5]. The effect of this partial registration is to deform the extremities of the X-ray image to the boundary shape of the MRI contrast projection.

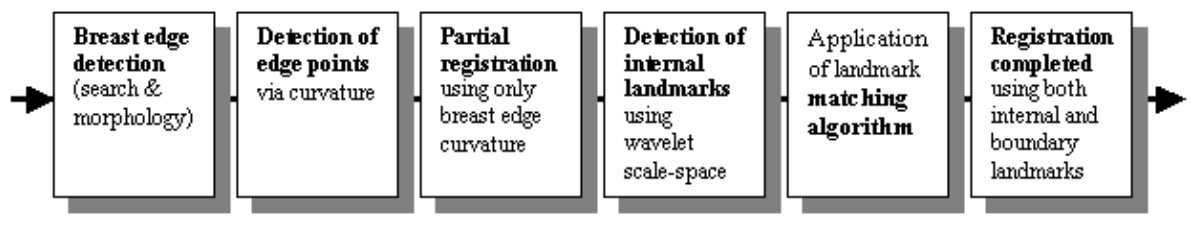

Fig. 1. Overview of X-ray to MRI contrast projection registration process

A second registration is performed based on the selection of internal landmarks using a wavelet-based feature detector. Internal landmarks in both the X-ray image and the MRI projection are matched using the following criteria:

- Scale localisation (i.e. landmarks of similar scale are matched)

- Orientation (via Principal Component Analysis) 
- Relative motion between the undeformed and the partially registered (via curvature points) data

- Neighbourhood localisation in the partially registered images

The second registration uses the combination of matched internal landmarks and boundary points (selected on the basis of curvature) to complete the registration. The registration technique adopted to date is thin plate spline warping [6]. This landmarkdriven approach is appropriate in applications where we do not necessarily have an intensity correlation between the MRI contrast projection and the X-ray data. It also enables us to perform the partial registration using only boundary points, which is particularly critical in the case where the breast has undergone significant involution ${ }^{1}$ and there may be no landmarks or intensities suitable for controlling the internal deformation of the registration process. An example of this 2-stage registration process is shown in Figure 2.
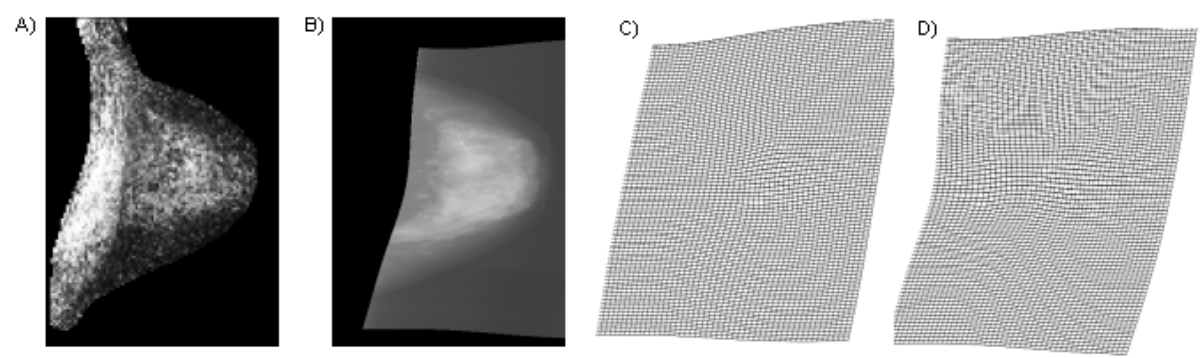

Fig. 2. A) shows the functional projection of the MRI volume in the cranio-caudal plane. $B$ ) shows the deformation of the cranio-caudal X-ray of the breast to the MRI contrast volume projection (cropped). $C$ ) and $D$ ) show the deformation grid of the partially and fully registered images. The increased level of complexity in the deformation of $D$ ) can be attributed to the internal landmarks used to complete the registration process

In the following sections, the main components of the registration process are discussed, namely the boundary curvature measure, internal landmark detection and the use of approximating thin-plate splines.

\section{Breast Boundary Feature Detection}

The detection of suitable landmarks for boundary deformation between the X-ray mammogram and the MRI volume projection first involves segmenting the breast

\footnotetext{
${ }^{1}$ The term "involution" refers to the transformation of ductal or parenchymal breast tissue into fat. The incidence of involution increases sharply at the onset of the menopause. The transradiance of fat relative to ductal/parenchymal tissue is the reason screening is typically restricted to women over the age of 50, a heuristic approximation to the age of onset of the menopause.
} 
edge. This is performed using a combination of an intensity-based search, mathematical morphology to smooth the breast edge (closing operator) and finally a B-spline interpolation of the segmented edge for curvature parameterisation [4]. Figure 3 shows the correlation between the spline-parameterised curvature and anatomical landmarks such as the nipple and axilla.
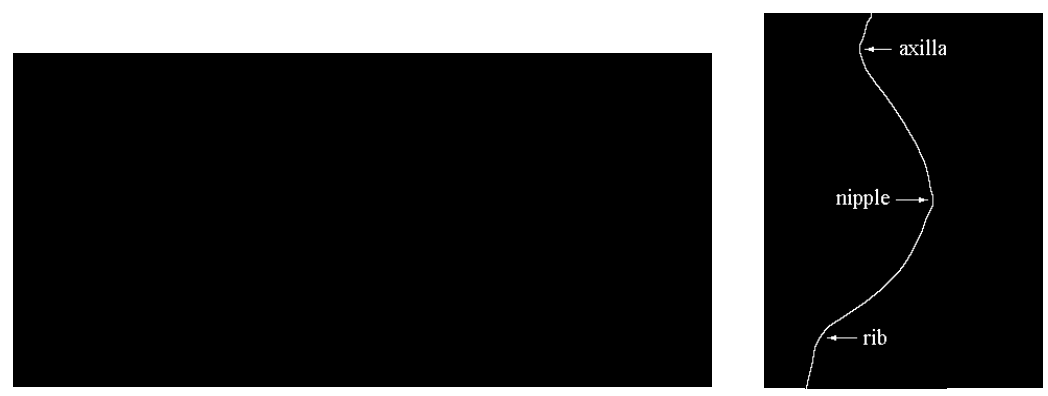

Fig. 3. An example curvature profile and corresponding anatomical landmarks. The curvature profile is separated into positive (dark) and negative (light) in order to locate the rib/axilla and nipple respectively

These curvature-selected points form the basis of the first step in the registration process, which effectively ensures that the boundary of the X-ray image is warped onto the MRI volume projection. However, this is not a true correction for breast compression and therefore internal landmarks are required to complete the compensation for complex internal soft tissue deformation. This technique has been reliably applied to dozens of mammograms [4,7] and is a sensible approach because while there may be slight changes of compression in successive mammograms (particularly around the time of the menopause), the breast outline shape remains consistent.

\section{Partial Registration}

Given the edge-localised curvature landmarks, the first registration step is performed (refer to Figure 1) using thin-plate spline approximation. In the thin-plate spline interpolation approach a set of $\mathrm{n}$ landmarks $\left(p_{i}, q_{i}\right)$ is used, where $p_{i}=\left(x_{i l}, y_{i 1}\right)$ are the co-ordinates of the landmarks of the first image and $q_{i}=\left(x_{i 2}, y_{i 2}\right)$ of the second. The interpolation problem is to find the transformation $f$ that fulfils the condition $f\left(p_{i}\right)=q_{i}$ for $i=1, \ldots, n$. and minimises a functional $J(\mathrm{f})$. $J(\mathrm{f})$ can be separated in $d=2$ (where $\mathrm{d}$ is the dimension of the images) problems for each component $f$ of $\mathrm{f}$. Thus for $d=2$ and $m=2$ (the number of derivatives used we get [6]:

$$
J_{2}^{2}(f)=\iint_{R^{2}}\left\{\left(\frac{\partial^{2} f}{\partial x^{2}}\right)^{2}+\left(\frac{\partial^{2} f}{\partial y^{2}}\right)^{2}+2 \cdot\left(\frac{\partial^{2} f}{\partial x \cdot \partial y}\right)^{2}\right\} d x d y
$$


This functional is also known as the bending energy of the deformation. In thin-plate spline theory, the desired function $f$ is the solution of the biharmonic equation $\Delta^{2} f=0$. It can be deformed in certain parts obeying the function $f(x, y)$, as long as the displacements are small and conforms to the minimum bending energy configuration of $f(x, y)$.

\section{Wavelet-Based Feature Detection}

To complete the registration internal landmarks are required to compensate for the complexity of the soft-tissue deformations. A scale-space approach was chosen, based on tensor wavelet packets $[8,9]$. Wavelet packet decompositions are particularly useful linear superpositions of wavelets that result in large "libraries" of functions that have specific frequency and spatial localisations [10]. The scale space construction follows a conventional dyadic decomposition using quadrature filters (QFs), as expressed in equation(s) 2 .

$$
\begin{array}{ll}
\psi_{0} \triangleq L_{f} \psi_{0} ; & \int_{\mathrm{R}} \psi_{0}(p) . d p=1, \\
\psi_{2 n} \triangleq L_{f} \psi_{n} ; & \psi_{2 n}(p)=\sqrt{2} \sum_{j \in Z} l_{f}(j) \psi_{n}(2 p-j), \\
\psi_{2 n+1} \triangleq H_{f} \psi_{n} ; & \psi_{2 n+1}(p)=\sqrt{2} \sum_{j \in Z} h_{f}(j) \psi_{n}(2 p-j),
\end{array}
$$

where $\psi_{0}$ is the mother wavelet and $\psi_{2 n}$ and $\psi_{2 n+1}$ are the resulting wavelet subspaces after convolution and decimation with the low-pass/high-pass equivalent QFs $h_{f}, l_{f}$ for pixel $p$ (in one pixel vector). To date, we have used the Coiflet wavelet bases as they feature good spatial localisation (i.e. edge preserving), have compact support [11] and are morphologically relevant to detecting small regions such as microcalicifications.

After wavelet decomposition the scale-space is completed by using an information cost function in the context of a "best basis" algorithm [12] to order wavelet coefficients in such a way that the various subspaces used for the decomposition are ranked by information content:

evaluated as:

$$
\|f-g\|_{L_{2}(I)}^{2}+\lambda\|g\|
$$

$$
\|f-g\|_{L_{2}(I)}^{2} \stackrel{\Delta}{=}\left(\int_{I}|f(p)-g(p)|^{2} d x\right)^{1 / 2}
$$

where $f(p)$ is the original image pixel vector and $g(p)$ is the reconstructed image pixel vector for a given wavelet subspace (packet) in $L_{2}$ with respect to the chosen cost function. In this case, we use a second-order approximation to entropy that is relatively invariant to image noise statistics [13]. Each wavelet subspace (filter superposition) is then cumulatively adjointly convolved, in order, with respect to the best-basis assessment of the decomposition. The result is a "stack" of reconstructions from minimum to maximum information content (dependent on the cost function). This analysis is used as the basis of segmentation, similar to the "extremum stack concept" [14], in conjunction with some contour refinement to compensate for smoothing [15]. 


\section{Final Registration}

The last step in the registration process is to include both the boundary curvaturebased landmarks and detected internal landmarks in the registration process (as outlined in part 3). In addition to the matching criteria discussed previously, the scale localisation information for each internal landmark is used to control the $\sigma_{i}^{2}$ terms in equation (5). This has the effect of applying a confidence measure to a landmark and adjusting the corresponding level of local deformation caused by each feature point. In this way, features with low-levels of saliency influence the internal deformation to a lesser extent. The implementation involves minimizing the functional [16]:

$$
J_{\lambda}(f)=\sum_{i=1}^{n} \frac{\left|\mathrm{q}_{\mathrm{i}}-\mathrm{p}_{\mathrm{i}}\right|^{2}}{\sigma_{i}^{2}}+\lambda \cdot J_{2}^{2}(f)
$$

where $\lambda$ is the regularisation parameter that controls the amount of smoothness in the deformation and $\sigma_{i}^{2}$ are the uncertainty terms.

\section{Clinical Assessment}

In an initial study, five patient datasets demonstrating a wide range of pathology were used to test the procedure ${ }^{2}$. The objective is to gauge how closely a correspondence can be made between the locations of pathology in the X-ray images with the 3D MRI volume representation. Cases were deliberately chosen to encompass a wide range of tissue biomechanical properties. For example, a large, dense ductal carcinoma (as shown in Figure 4, for example) has very different deformation properties than localised fibrosis (a brief numerical assessment of these five cases is presented in Table 1).

For the purpose of presenting the visualisation results shown in this paper, two extreme cases were chosen. The first features a dense circumscribed tumour in a highly involuted breast. This tumour is very large ( 4.5 centimetres in diameter) but is quite poorly differentiated with a highly fibrous epithelium. This is an interesting case because the effects of compression have quite clearly deformed the shape of the tumour in the mammogram. Figure 4 shows a shape comparison (via our scale-space segmentation) between the mammograms, the MRI contrast projection and the registered images.

The registration shown in Figure 4 is quite good, although the lack of large numbers of internal landmarks has perhaps oversimplified the internal deformation. For further comparison, an averaged centroid coordinate has been taken from both views and used to reconstruct the shape of the "uncompressed" breast using the technique outlined in [17]. This can be compared visually with a segmentation of the tumour in the MRI breast volume using a 3D implementation of the Hayton-Brady pharmacokineticbased segmentation [3]. This comparison can be seen in Figure 5.

2 The patients were being assessed at the Oxford MRI clinic and volunteered their data in accordance with the local ethical policy. 

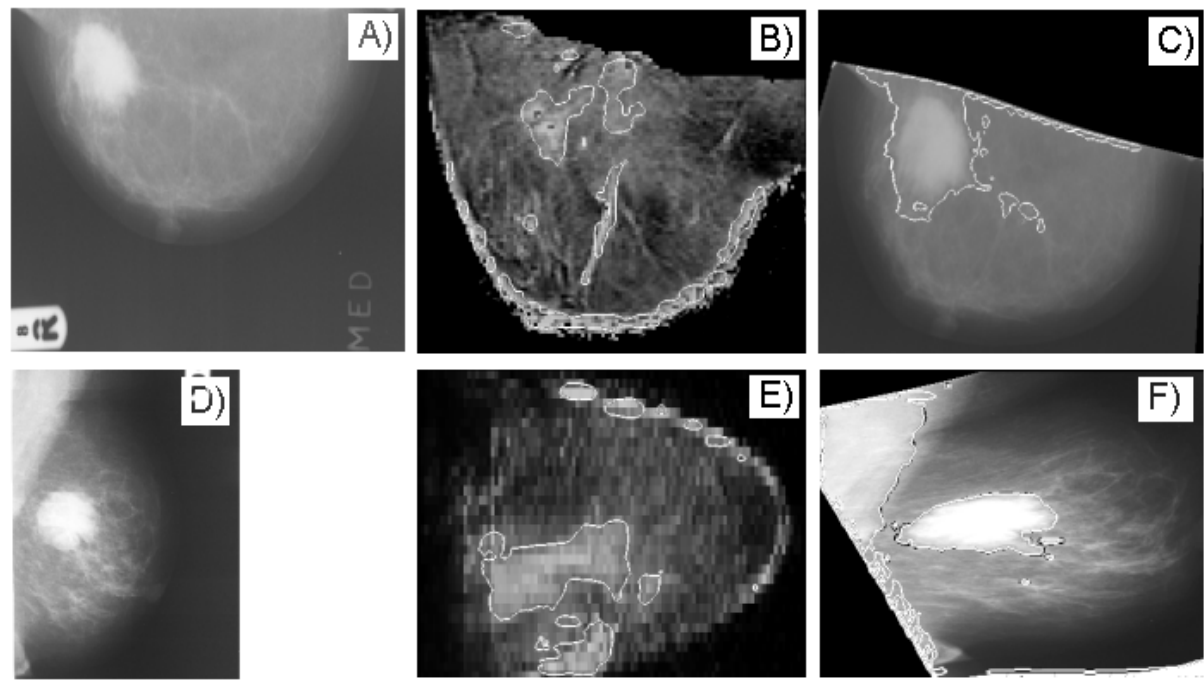

Fig. 4. A) and D) are the original CC and ML X-rays (scaled). B) and E) are MRI enhancement projections showing segmented regions of interest. $C$ ) and $F$ ) are the CC and ML mammograms registered to the MRI projection images with regions of interest segmented
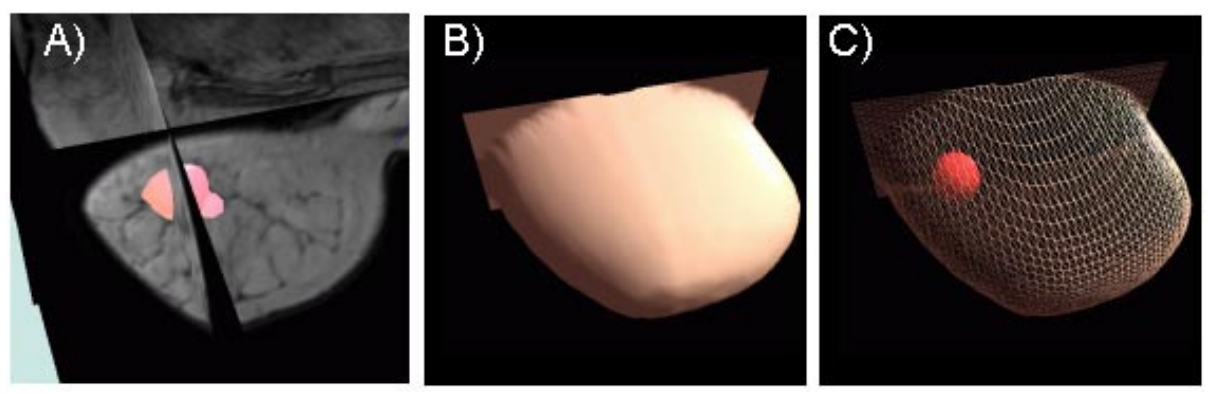

Fig. 5. A) shows the pharmacokinetic segmentation of the tumour in $3 \mathrm{D}$ from the contrastenhanced MRI acquisition. Figure $B$ ) shows the reconstructed shape of the breast from the registered X-ray projections. $C$ ) shows the location of the tumour in the same registered reference frame

The second case highlights one of the most important applications of this research. It has been observed that certain shapes and distributions of microcalcifications are indicative of ductal carcinoma. However, many cases of DCIS don't enhance particularly well under CE-MRI, nor are microcalcifications visible. However, for surgical planning, it would be useful to be able to correlate the locations of the microcalcification to a 3D position in the breast. Figure 6 shows an example of a patient with severe ductal ectasia ("exploded" ducts) and two coarse calcifications located near the nipple and top-end of the parenchymal tissue. Using exactly the same technique as for the first case, the location of the calcifications in the 3D MRI volume was found. 


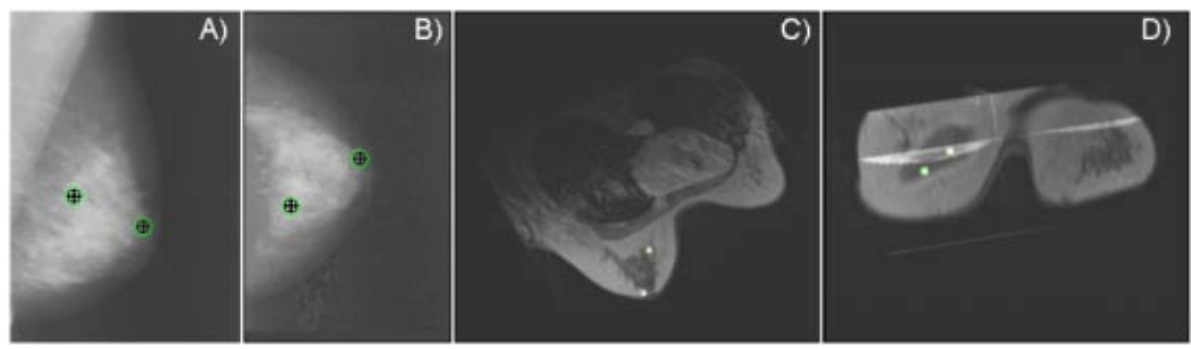

Fig. 6. A) and $B$ ) are the original $\mathrm{ML}$ and $\mathrm{CC}$ mammograms (respectively). The calcifications have been circled for clarity. $C$ ) and $D$ ) show the location of two micro-calcifications in the resliced MRI volume after registration

Previous work has enabled a partially compensated 3D spatial reconstruction of microcalcifications [17]. However, our approach enables regions of interest (including calcifications) to be compared against a true uncompressed representation, rather than a simulated and partially uncompressed representation. Table 1 contains a summary of the five cases used for this study. In all cases, clinician-identified regions of interest we registered to within $+/-4$ voxels (approx. 4mm using our current MRI volume sequence).

Table 1. The approximate error (with respect to the MRI volume) of each of the cases used in this study

\begin{tabular}{|c|c|c|c|c|}
\hline Case & Condition & CC Centroid Error & MI Centroid Error & Avg. Error \\
\hline 1. & Small opacity, infiltrating carcinoma & -3.5 Voxels (avg) & -2.5 Voxels (avg) & 3 Voxels \\
2. & Fibroglandular nodule $(1.5 \mathrm{~cm})$ & +1.5 Voxels & -4 Voxels & 2.75 Voxels \\
3. & Large, dense circumscribed tumour & +3 Voxels & +5.5 Voxels & 4.25 Voxels \\
(ductal carcinoma) & $\begin{array}{c}\text { Multiple microcalcifications } \\
\text { 5. }\end{array}$ & -1 Voxe1 (avg) & -3 Voxels (avg) & 2 Voxels \\
& $\begin{array}{c}\text { Spiculated opacity associated with large } \\
\text { lymph node }\end{array}$ & -2 Voxels & +5 Voxels & 3.5 Voxels \\
\hline
\end{tabular}

\section{Discussion and Further Work}

As with most applications of non-rigid registration, the success of the process outlined in this paper is quite difficult to evaluate. Table 1 shows the estimated error in localising the centroid of the various pathologies in the two views. Clearly, in the case of calcifications, this error estimation is difficult as surrounding tissue, rather than the feature itself must form the basis of the assessment. Additionally, the accuracy of the ML registration must be somewhat less as the cross-plane projection of the MRI volume is limited by the number of slices in the volume data (in this case 32). Further validation is necessary using phantoms, core-biopsy studies and a larger patient data set, however the preliminary results are quite encouraging particularly considering the extent of the deformation required for registration. 
Although the principal objective of this research is data fusion for pathology assessment, there are a number of related applications for this work. Firstly, MRI is the most commonly used uncompressed breast imaging modality. By registering Xray mammography (highly compressed) to MRI, the mammograms are effectively "decompressed". This provides an interesting framework for the study of breast tissue deformation under compression, with MRI effectively providing the uncompressed "gold standard". This would also provide independent validation of Highnam and Brady's h_int model of non-fatty breast anatomy computed from a mammogram [18].
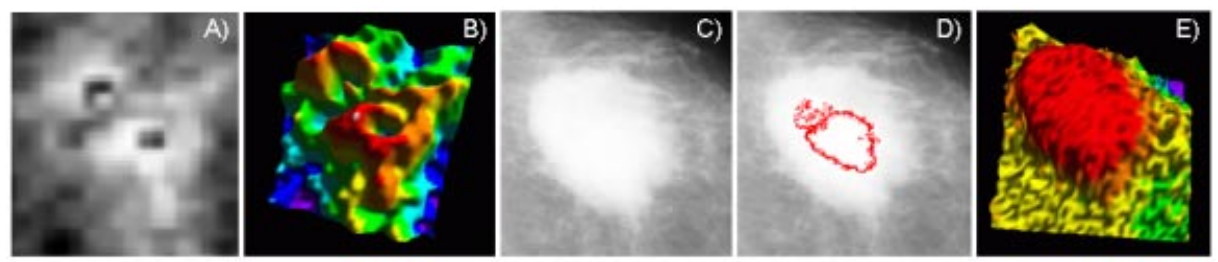

Fig. 7. This sequence of images shows a comparison between the functional projection of the pathology from the contrast-enhanced MRI data set and the X-ray structure in the CC mammogram of the example shown previously. A surface rendering $B$ ) of the (scaled) functional projection $A$ ) clearly shows two "rings" of contrast enhancement around a lowenhancement centre. This is quite typical of CE-MRI focal enhancement where the necrosis enhancement is relatively low compared with surrounding vascularity. Intensity equalisation and edge detection of the X-ray mass $C, D$ ) is consistent with the functional information as shown in $D$ ). $E$ ) is a surface rendering of $C$ ) for comparison with $B$ )

Finally, this registration approach may enable a functional representation to be compared with a structural (X-ray) understanding of the pathology. For example, in the case of the large tumour shown in Figures $5 \& 6$, it is interesting to compare the uptake of contrast in the tumour to the structure shown in the X-ray. This is shown in Figure 7 where the mass actually consists of two enhancing focal regions. With some simple thresholding and edge detection, it can also be shown that the same structure exists in the X-ray, although it would not be immediately apparent to a clinician.

\section{Acknowledgements}

C. Behrenbruch would like to acknowledge the Commonwealth Scholarship and Fellowship Program for his doctoral funding at Oxford University. The author would also like to acknowledge valuable discussions with Jerome Declerck (MVL) and Dermot Dobson (John Radcliffe Hospital). P. Armitage acknowledges the EPSRC for his post-doctoral grant (GR/M54995). J.M. Brady acknowledges EPSRC support for his senior fellowship. 


\section{References}

1. M. Lanyi, "Diagnosis and differential diagnosis of breast calcifications". Springer-Verlag, Berlin, 1986

2. K.G.A. Gilhuijs, "Automated verification of radiation treatment geometry", Universiteit van Amsterdam, Febo-druk, Enschede-Utrecht, The Netherlands, 1995

3. P. Hayton, J.M. Brady, L. Tarassenko, N. Moore, "Analysis of dynamic MR breast images using a model of contrast enhancement”, Medical Image Analysis, 1:3, April, 1997, Oxford University Press

4. K. Marias, J.M. Brady, et. al., "Registration and matching of temporal mammograms for detecting abnormalities", Medical Imaging Understanding and Analysis, Oxford 1999

5. K. Marias, C.P. Behrenbruch, J.M. Brady, "Robust Breast Edge Segmentation in Mammography”, Engineering Science Technical Report 19990805\#2, Oxford University, 1999

6. F.L. Bookstein, Principal Warps: "Thin-Plate Splines and the Decomposition of Deformations", IEEE Transactions on Pattern Analysis and Machine Intelligence, 11:6, pp 567-585, June 1989.

7. K. Marias, C.P. Behrenbruch, et. al., "Multi-scale Landmark Selection for Improved Registration of Temporal Mammograms", IWDM (International Workshop in Digital Mammography) 2000, Toronto, Canada, Elsevier, June 2000.

8. R.R. Coifman, Y. Meyer, S.R. Quake, M.V. Wickerhauser, "Signal processing and compression with wavelet packets". In Meyer and Roques [9], pp 77-93, 1992

9. Y. Meyer, S. Roques, (editors), "Progress in Wavelet Analysis and Applications". Proceedings of the International Conference "Wavelets and Applications", Toulouse, France, 8-13 June 1992. Observatoire Midi-Pyrénées de l'Université Paul Sebatier, Editions Frontieres

10. I. Daubechies, "Orthonormal bases of compactly supported wavelets". Communications on Pure and Applied Mathematics, XLI: pp 909-996, 1988

11. I. Daubechies, "Ten lectures on wavelets", $C M B S$, SIAM, 61, pp 258-261, 1994

12. R.R. Coifman, \& M.V. Wickerhauser, "Entropy based algorithms for best basis selection", IEEE Transactions on Information Theory, 32, pp 712-71, 1992

13. G.J.M. Parker, J.A. Schnabel, G.J. Barker, "Nonlinear Smoothing of MR Images Using Approximate Entropy - A Local Measure of Signal Intensity Irregularity". Technical Report, NMR Research Unit, Institute of Neurology, University College London, 1998

14. J. Koenderink,, "The structure of images", Biological Cybernetics, vol. 50, pp 363-370, 1984

15. S.C. Zhu, A. Yuille, "Region Competition: Unifying Snakes, Region Growing, and Bayes/MDL for Multiband Image Segmentation”, PAMI 18:9, September 1996, pp. 884900.

16. K. Rohr et. al., "Point-based elastic registration of medical image data using approximating thin-plate splines", Lecture Notes in Computer Science 1131, Springer, pp. 297-306, 1996

17. M. Yam, J.M. Brady, et. al., "Reconstructing microcalcification clusters in 3-D using a parameterized breast compression model", Computer Assisted Radiology and Surgery (CARS), Paris 1999.

18. R.P. Highnam and J.M. Brady, "Mammographic Image Processing", Kluwer Academic Press, 1999 